\title{
Características Cefalométricas en Jóvenes con Oclusión Normal y Perfil Armónico en Población Chilena
}

\author{
Cephalometric Characteristics in Young Individuals with Normal \\ Occlusion and Harmonic Profiles in Chilean Population
}

Joaquín Montt Rodriguez; María Paz Miquel Valdés* \& Rodrigo Oyonarte Weldt**

MONTT, R. J.; MIQUEL, V. M. P. \& OYONARTE, W. R. Características cefalométricas en jóvenes con oclusión normal y perfil armónico en población chilena. Int. J. Morphol., 33(1):237-244, 2015.

RESUMEN: Las normas cefalométricas facilitan el diagnóstico y planificación terapéutica en ortodoncia, siendo estas obtenidas primariamente de población caucásica adulta. Dichas normas variarían entre etnias siendo escasa la información respecto de las de población chilena en crecimiento. Se obtuvo una muestra de 48 telerradiografías de perfil de individuos en crecimiento ( 23 mujeres y 25 hombres) con oclusión normal y perfil armónico de los registros de la Universidad de los Andes tomados entre Enero y Julio del año 2012 , incluyendo 414 individuos. Las radiografías fueron analizadas cefalométricamente, obteniéndose valores de parámetros verticales y sagitales en tejidos blandos y duros. Se obtuvieron estadísticas descriptivas, y las diferencias entre sexos se analizaron con la prueba t de Student. Al compararse con normas caucásicas, los sujetos de la muestra tienen una tendencia a la Clase II esqueletal, por una mandíbula retrognática, eje facial abierto, y biprotrusión incisiva. También mostraron labios superiores e inferiores protruidos respecto de las normas caucásicas. Existen diferencias entre las medidas cefalométricas de la muestra y las de las normas internacionales, manifestadas a nivel mandibular, dentario y labial.

PALABRAS CLAVE: Normas cefalométricas; Cefalometría en tejidos duros; Cefalometría en tejidos blandos; Normas cefalométricas chilenas.

\section{INTRODUCCIÓN}

Las anomalías dentomaxilares son altamente prevalentes (Soto \& Tapia, 2007) y presentan una manifestación de intensidad variable. Éstas tienen implicancias anatómicas, funcionales y estéticas a nivel dentofacial que frecuentemente justifican su tratamiento en base a decisiones clínicas apoyadas en la valoración diagnóstica de la telerradiografía de perfil mediante la cefalometría. Así, el uso de normas cefalométricas permite la comparación de un paciente con un grupo de referencia normal que define las medidas cefalométricas ideales, basadas en factores tales como edad, sexo y raza. No obstante lo anterior, las normas cefalométricas caucásicas son comunmente utilizadas en diagnóstico y planificación terapéutica ortodóncica sobre individuos de diversas etnias.

Se ha reportado que la norma cefalométrica de un grupo puede no ser apropiada para otros grupos raciales (Vela et al., 2011; Hassan, 2006; Jain \& Kalra, 2011). En efecto, en Latinoamérica se han realizado estudios que demuestran diferencias cefalométricas respecto de las normas caucásicas tanto en tejidos duros como blandos (Scavone et al., 2008;
Swlerenga et al., 1994). Así, individuos de distinto origen étnico se favorecen de ser diagnosticados y tratados de acuerdo a normas propias de su respectiva etnia, pues el uso de normas inapropiadas a la población tratada puede llevar a errores diagnósticos y terapéuticos.

El desarrollo craneofacial implica una evolución de las características faciales en las que se producen variaciones cefalométricas tanto lineales como angulares, a nivel de tejidos blandos y duros (Mamandras, 1988; McNamara, 1984). La información es escasa respecto a las características de individuos mestizos chilenos con oclusión normal (Clase I de Angle) y perfil armónico en dentición mixta y permanente joven, los que habitualmente son evaluados en base a normas caucásicas.

El objetivo fue describir las características cefalométricas en tejidos duros y blandos en una muestra de individuos en crecimiento de ambos sexos con oclusión normal y perfil armónico obtenida en la comuna de San Bernardo, Región Metropolitana, Chile.

* Cirujano-Dentista, Práctica Privada, Santiago, Chile.

** Cirujano-Dentista, Ortodoncista, Profesor Asociado Facultad de Odontología, Universidad de los Andes, Santiago, Chile. 


\section{MATERIAL Y MÉTODO}

Se realizó un estudio de tipo transversal sobre registros radiológicos de pacientes atendidos en el Centro Universitario de Especialidades Médicas de la Universidad de Los Andes, San Bernardo, Chile durante 2012. Se incluyeron a los individuos según los criterios descritos en la Tabla I.

La muestra se estableció ejecutando un muestreo consecutivo de los registros de pacientes con radiografías bite-wing bilateral, panorámica y telerradiografía de perfil, con sus respectivas fichas clínicas de derivación al servicio de radiología, ejecutadas por ortodoncistas. Se incluyó a todos los individuos radiografiados en el servicio con registros completos entre Enero y Julio de 2012 (Fig. 1). Las radiografías fueron obtenidas por un operador único en el mismo equipo radiológico (Ortophos XG5, Sirona $\left.{ }^{\circledR}\right)$. Las telerradiografías se obtuvieron en posición natural de la cabeza y con los dientes en oclusión.

El material radiológico utilizado fue obtenido con el consentimiento informado de los apoderados de los pacientes, el que autoriza el uso de los registros con fines académicos de investigación. A su vez, la investigación contó con la aprobación del comité de ética de la Facultad de Odontología de la Universidad de los Andes.

Una vez obtenida la muestra se realizó el análisis cefalométrico por un ortodoncista experimentado (RO), previamente calibrado en la ubicación de estructuras cefalométricas, mediante el coeficiente de correlación intraclase a partir de un modelo multinivel utilizando el programa STATA® 11.

Las telerradiografías de perfil fueron analizadas utilizando el programa cefalométrico Dolphin ${ }^{\circledR} 11.5$ Premiun. También se evaluó la maduración esqueletal de los sujetos, con el método de maduración vertebral cervical (MVC) de Baccetti et al. (2005), por el mismo operador.

Las variables analizadas incluyeron edad, sexo, tipo de dentición, resalte (overjet), sobremordida vertical (overbite), maduración vertebral cervical (MCV), ángulo SNA, ángulo SNB, ángulo ANB, apreciación de Wits, ángulo Sn-GoGn, eje facial (Na-Ba y Pt-Gn), ángulo del incisivo maxilar con el plano palatino (ENA-ENP), ángulo del incisivo mandibular y el plano mandibular (Go-Me), distancia del incisivo maxilar a la línea A-po y distancia del incisivo mandibular a la línea A-po, posición de labios superior e inferior (Ls- SnPog' y Li- SnPog'), ángulo nasolabial (Cm-Sn-Ls), profundidad del surco mentolabial (Si- LiPog'), ángulo facial (N'-Sn-Pog'), y exposición de incisivo maxilar (Stms- U1 tip) (Figs. 2 y 3). La medición de las variables cefalométricas fue realizada en base a las descripciones de Steiner (1953), Ricketts (1960), Tweed (1946), McNamara, Jacobson (1975), Bishara (1985), Uribe Restrepo (2007) y Legan \& Burstone (1980).

Tabla I. Criterios de inclusión para el estudio cefalométrico en jóvenes con oclusión normal.

1. Clase I de Angle.

2. Ausencia de mordida cruzada o invertida.

3. Apiñamiento o espaciamiento incisivo ausente o leve (visto en la panorámica como sobreproyección leve o ausente de caras interproximales o diastemas mínimos entre caras proximales).

4. Dentición permanente o mixta con incisivos centrales totalmente erupcionados.

5. Ausencia de pérdida de espacio interproximal por caries o por pérdida prematura de dientes temporales.

6. Ausencia de tratamiento ortodóncico previo.

7. Perfil armónico (equilibrio de los tercios faciales).

8. Ausencia de mal hábito de succión de dedo y chupete.

9. Resalte (overjet) mayor o igual a 1 y menor o igual a $4 \mathrm{~mm}$.

10. S obremordida vertical (overbite) mayor o igual a 1 y menor o igual a $4 \mathrm{~mm}$.

11. B recha (gap) labial menor o igual a $4 \mathrm{~mm}$. 


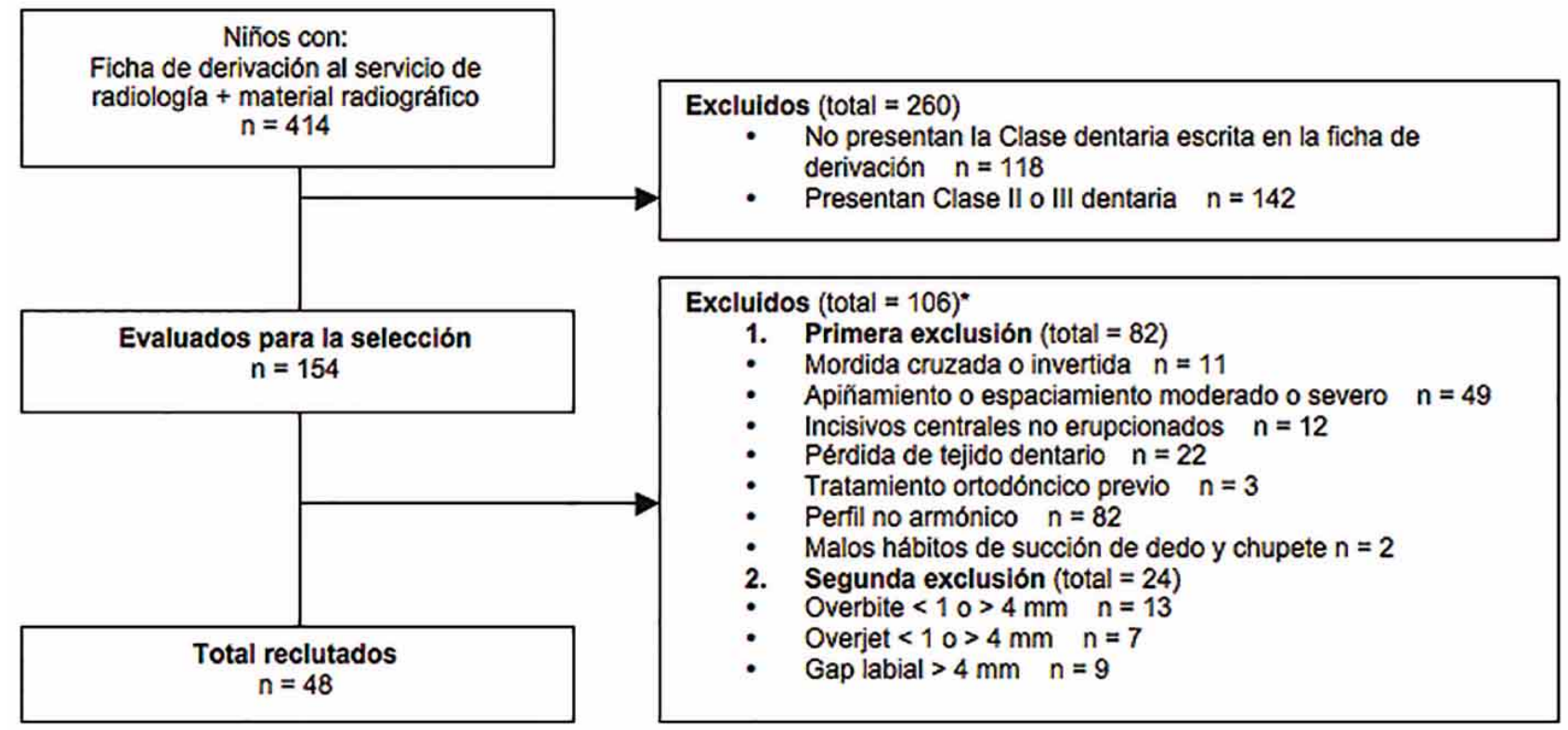

Fig. 1. Diagrama de flujo de la determinación de la muestra.

* La letra "n" indica el número de pacientes; Los valores no son excluyentes, con pacientes que cumplen con más de un criterio de exclusión.

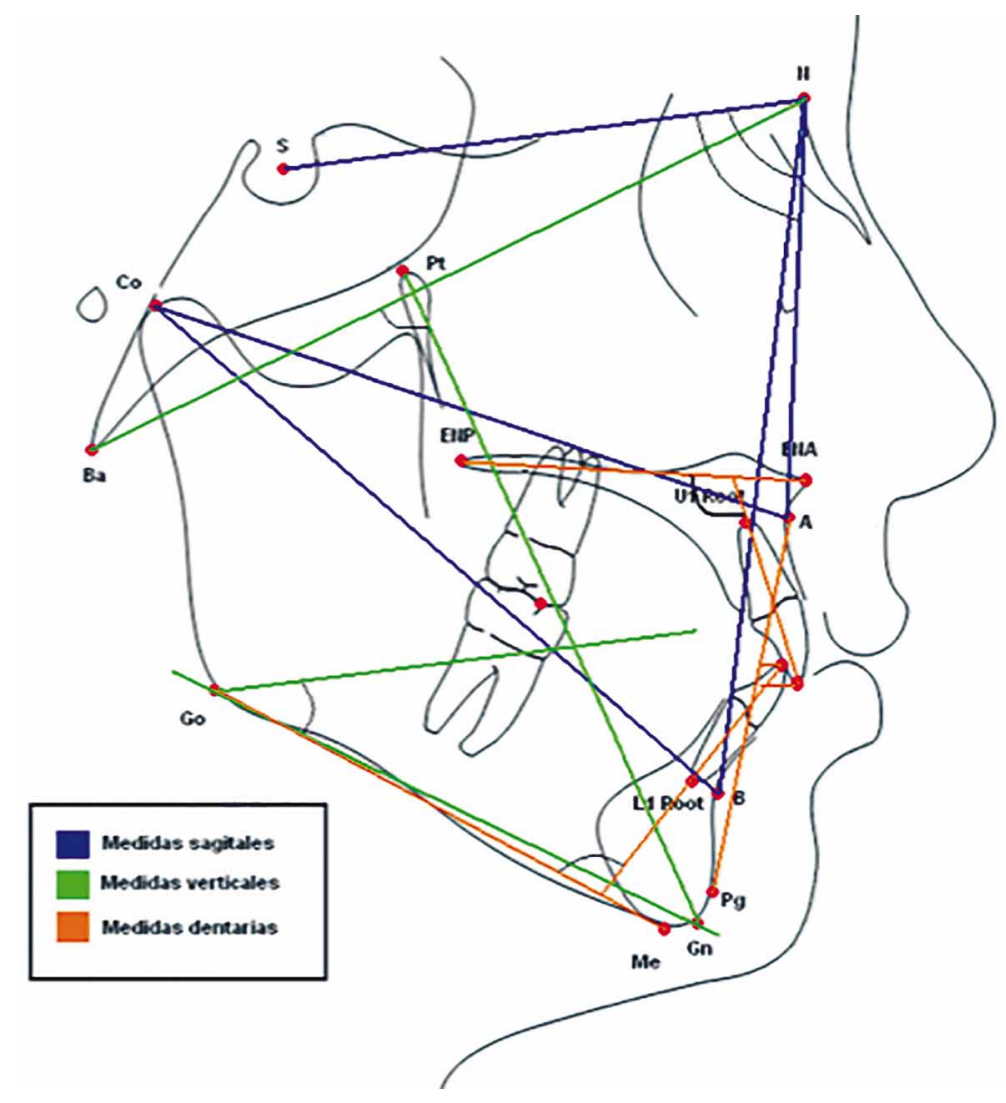

Fig. 2. Figura que muestra los trazados cefalométricos de tejidos duros. 


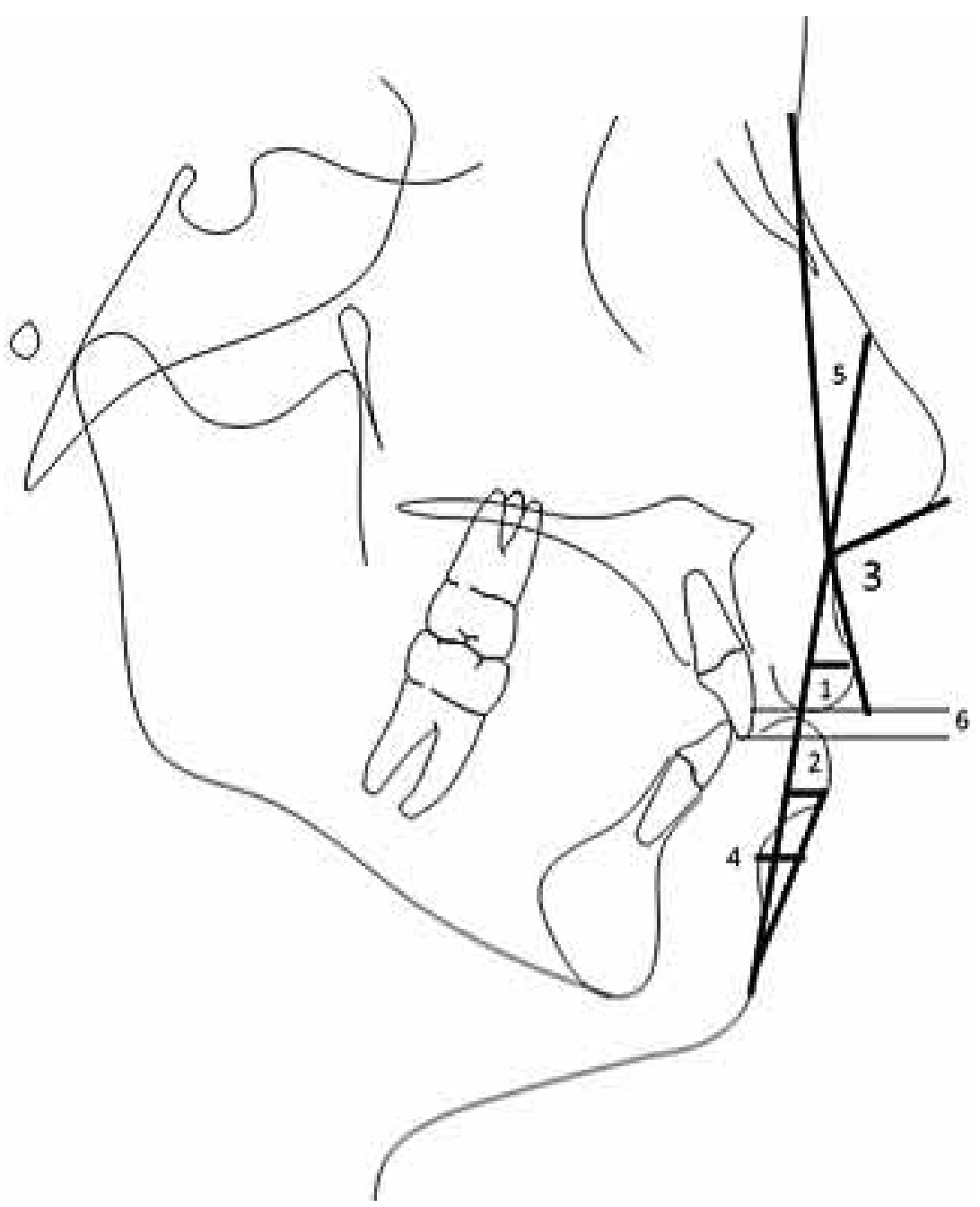

Fig. 3. Figura que muestra los trazados cefalométricos de tejidos blandos.

El análisis estadístico se realizó con el programa STATA 11®. Se obtuvieron estadísticas descriptivas en torno a sexo, edad, tipo de dentición, resalte y sobremordida vertical. La etapa de maduración cervical se analizó en base a frecuencia y porcentaje respecto a cada etapa de maduración cervical de la muestra según la edad y el sexo, con sus respectivos porcentajes.

Respecto a las medidas cefalométricas, se obtuvieron estadísticas descriptivas, luego de evaluar la normalidad de cada variable mediante la prueba de Shapiro - Wilk. También se obtuvieron estadísticas descriptivas de las medidas cefalométricas según sexo. Para evaluar diferencias entre promedios de las medidas cefalométricas para los distintos sexos se utilizó la prueba t de Student. Se exploraron a su vez la existencia de diferencias entre los promedios de la muestra y las normas caucásicas. Para describir la existencia de diferencias entre los promedios de las medidas cefalométricas encontradas en la muestra y la norma utilizada en pacientes caucásicos, se realizaron tablas comparativas entre el valor promedio obtenido en la muestra para la variable cefalométrica y su respectiva desviación estándar y los obtenidos por las normas publicadas.

\section{RESULTADOS}

Luego de analizar los datos de los 414 pacientes que conformaron el conjunto de individuos con registros clínicos y radiográficos completos, se obtuvo una muestra 48 individuos $(25$ hombres y 23 mujeres) de entre 8 y 15 años (promedio de 10,8 años de edad), mayormente en dentición permanente.

La Tabla II contiene las estadísticas descriptivas para cada una de las medidas cefalométricas estudiadas en las telerradiografías de perfil y una comparación de los valores de promedios de las medidas obtenidas de la muestra del estudio y los valores promedio de varios autores con muestras de pacientes caucásicos. Los resultados indican que los valores del ángulo SNA, el ángulo SN-GoGn y la inclinación de los incisivos maxilares y mandibulares se asemejan a las normas caucásicas.

Respecto a los valores que presentaron diferencias con la norma se encuentran el ángulo SNB $\left(77,8 \pm 3,1^{\circ}\right)$, el cual está disminuído respecto al valor de la norma establecida por Steiner; el valor para el ángulo ANB $\left(4 \pm 1,8^{\circ}\right)$ está aumentado con respecto al valor de la norma de Steiner, descrito en $2 \pm 2^{\circ}$. En cuanto al eje facial (NaBa-PtGn) los pacientes de la muestra presentaron un valor de $86,6 \pm 4,2^{\circ}$, el cual está disminuido con respecto a la norma publicada por Ricketts.

Dentro de las medidas dentarias, las mediciones de posición de los incisivos maxilares y mandibulares, U1Apo y L1Apo $(7,1 \pm 1,7 \mathrm{~mm}$ y $3,7 \pm 1,5 \mathrm{~mm}$, respectivamente) se encuentran considerablemente aumentadas respecto a las normas publicadas por Ricketts, indicando biprotrusión incisiva.

Con respecto a los tejidos blandos se constatan diferencias entre los promedios de las medidas de la muestra con las normas de Legan \& Burstone en 
Tabla II. Medidas de tendencia central y de dispersión de las variables cefalométricas y su diferencia con la norma caucásica.

\begin{tabular}{|c|c|c|c|c|c|c|c|}
\hline \multirow[t]{2}{*}{ Variables } & & $\begin{array}{c}\text { Muestra } \\
n=48\end{array}$ & \multirow[b]{2}{*}{ P50 } & \multirow[b]{2}{*}{ RIC } & \multirow[b]{2}{*}{ Rango } & \multirow{2}{*}{$\begin{array}{c}\begin{array}{c}\text { Normas } \\
\text { caucásicas }\end{array} \\
\text { Promedio } \pm \text { DE }\end{array}$} & \multirow[b]{2}{*}{ Dif. } \\
\hline & & Promedio \pm DE & & & & & \\
\hline \multirow[t]{4}{*}{ I. Sagitales } & SNA $\left(^{\circ}\right)$ & $81,9 \pm 3,2$ & 81,7 & 4,3 & $75,2-90,2$ & $82 \pm 2$ & (-) 0,1 \\
\hline & $\mathrm{SNB}\left({ }^{\circ}\right)$ & $77,8+3,1$ & 78 & 3,8 & $70,6-87,6$ & $80+2$ & (-) 2,2 \\
\hline & $\mathbf{A N B}\left({ }^{\circ}\right)$ & $4,0+1,8$ & 4 & 2,9 & $0-7,9$ & $2+2$ & 2 \\
\hline & Wits $(\mathbf{m m})$ & 0,8 & $-1,3$ & 3,1 & $-5,7-3,4$ & --- & --- \\
\hline \multirow[t]{2}{*}{ II. Verticales } & SN - GoGn $\left({ }^{\circ}\right)$ & $31,8+4,7$ & 31,8 & 6,4 & $22,5-43,4$ & $32+2$ & (-) 0,2 \\
\hline & Eje facial $\left({ }^{\circ}\right)$ & $86,6+4,2$ & 86,5 & 5,5 & $75,8-98,5$ & $90+3$ & (-) 3,4 \\
\hline \multirow[t]{4}{*}{ III. Dentarias } & $\mathbf{U} 1$ - PP $\left(^{\circ}\right)$ & $109,9 \pm 5,1$ & 110 & 7,6 & $97,6-119,6$ & $110 \pm 5$ & (-) 0,1 \\
\hline & $\mathbf{L} 1-\operatorname{PM}\left(^{\circ}\right)$ & $93,9+4,9$ & 93,1 & 8,1 & $84,7-104,4$ & $90+3$ & 3,9 \\
\hline & U1 - Apo (mm) & $7,1+1,7$ & 6,8 & 2,3 & $4,6-11$ & $3,5+2$ & 3,6 \\
\hline & L1 - Apo (mm) & $3,7 \pm 1,5$ & 3,3 & 2,3 & $1,5-7,3$ & $1 \pm 2$ & 2,7 \\
\hline \multirow{7}{*}{ IV. $\frac{\text { Tejidos }}{\text { Blandos }}$} & Ls- SnPog'(mm) & $5,7+1,5$ & 5,4 & 2,1 & $3,1-8,6$ & $3 \pm 1$ & 2,7 \\
\hline & Li-SnPog' (mm) & $4,3 \pm 1,5$ & 4,3 & 2,9 & $1,0-8,2$ & $2 \pm 1$ & 2,3 \\
\hline & Cm-Sn- Ls $\left(^{\circ}\right)$ & $107,1 \pm 8,1$ & 105,7 & 10,1 & $86,9-127,9$ & $102 \pm 8$ & 5,1 \\
\hline & GAP $(\mathbf{m m})$ & $1,0 \pm 1,2$ & 0,6 & 1,1 & $0,0-4,0$ & $2 \pm 2$ & 1 \\
\hline & $\mathbf{N}^{\prime}-\mathrm{Sn}-\mathrm{Pog}\left(^{\circ}\right)$ & $14,8 \pm 4,9$ & 15,4 & 6,8 & $3,8-27,4$ & $12 \pm 4,9$ & 2,8 \\
\hline & Si- LiPog'(mm) & $5,1 \pm 1,2$ & 5 & 1,3 & $2,7-8,0$ & $4 \pm 2$ & 1,1 \\
\hline & Stms- U1 tip (mm) & $3,4 \pm 1,6$ & 3,4 & 2,5 & $0,6-6,5$ & $2 \pm 2$ & 1,4 \\
\hline
\end{tabular}

DE= Desviación estándar, P50= Mediana, RIC= Rango intercuartílico.

todas las medidas, siendo mayores las diferencias en protrusión del labio superior (Ls- SnPog') e inferior (Li-SnPog').

En la Tabla III se presentan las diferencias entre sexos. A nivel de tejido duro, se observan diferencias estadísticamente significativas entre hombres y mujeres para el ángulo ANB, apreciación de Wits, la inclinación incisiva inferior (L1PM) y la posición del incisivo superior (U1Apo). En cuanto a las demás medidas registradas no hubo diferencias significativas entre sexos.

Tabla III. Diferencias entre sexos para variables cefalométricas

\begin{tabular}{|c|c|c|c|c|c|c|}
\hline \multirow{2}{*}{ Variable } & & \multirow{2}{*}{$\begin{array}{c}\text { Femenino } \\
\mathbf{n}=23\end{array}$} & \multirow{2}{*}{$\begin{array}{c}\text { Masculino } \\
\mathbf{n}=\mathbf{2 5}\end{array}$} & \multirow{2}{*}{$\begin{array}{l}\text { Diferencia } \\
\text { entre sexos }\end{array}$} & \multirow[b]{2}{*}{$p$ value } & \multirow{2}{*}{$\begin{array}{c}\text { Significación } \\
\text { estadística }\end{array}$} \\
\hline & & & & & & \\
\hline \multirow[t]{4}{*}{ I. Sagitales } & SNA & $81,7 \pm 3,1$ & $82,1 \pm 3,3$ & 0,4 & 0,679 & NS \\
\hline & SNB & $78,2 \pm 2,6$ & $77,5 \pm 3,5$ & 0,7 & 0,2 & NS \\
\hline & $A N B$ & $3,5+1,6$ & $4,5+1,9$ & 1 & 0,048 & $*$ \\
\hline & WITS & $-0,5$ & 1,7 & 1,7 & 0.013 & $*$ \\
\hline \multirow[t]{2}{*}{ II. Verticales } & SN-GoGn & $32,2 \pm 4,8$ & 31,5 & 0,7 & 0,613 & NS \\
\hline & Eje facial & $86,5+4,5$ & 86,7 & 0,2 & 0,854 & NS \\
\hline \multirow[t]{4}{*}{ III. Dentales } & U1PP & $109,9 \pm 4,9$ & 109,9 & 0 & 0,967 & NS \\
\hline & L1PM & $92,4+4,4$ & 95,2 & 2,8 & 0,046 & $*$ \\
\hline & U1Apo & $6,6+1,7$ & 7,5 & 0,9 & 0,01 & $*$ \\
\hline & L1Apo & $3,7 \pm 1,7$ & 3,8 & 0,1 & 0,634 & NS \\
\hline IV. Tejidos & Ls- SnPog' (mm) & $5,3 \pm 1,4$ & $6,0 \pm 1,6$ & 0,7 & 0,166 & NS \\
\hline \multirow[t]{6}{*}{ Blandos } & Li-SnPog' (mm) & $4,5 \pm 1,9$ & $4,2 \pm 1,9$ & 0,3 & 0,575 & NS \\
\hline & Cm-Sn- Ls $\left(^{\circ}\right)$ & $106,1 \pm 6,3$ & $108,0 \pm 9,4$ & 1,9 & 0,425 & NS \\
\hline & GAP $(\mathbf{m m})$ & $1,0 \pm 1,0$ & $0,9 \pm 1,3$ & 0,1 & 0,319 & NS \\
\hline & N'-Sn-Pog' $\left(^{\circ}\right)$ & $14,4 \pm 4,8$ & $15,2 \pm 5,1$ & 0,8 & 0,566 & NS \\
\hline & Si- LiPog' (mm) & $4,9 \pm 1,1$ & $5,3 \pm 1,3$ & 0,4 & 0,341 & NS \\
\hline & Stms- U1 tip & $3,1 \pm 1,5$ & $3,7 \pm 1,6$ & 0,6 & 0,228 & NS \\
\hline
\end{tabular}

$\mathrm{NS}=$ No significativa, $*=\mathrm{P}<0,05, * *=\mathrm{P}<0,01$. 


\section{DISCUSIÓN}

El presente estudio fue realizado con el objetivo de describir las características cefalométricas en tejidos duros y blandos en una muestra de individuos en crecimiento de ambos sexos con oclusión normal y perfil armónico obtenida en la comuna de San Bernardo, Región Metropolitana, Chile. Para ello se seleccionó una muestra obtenida de los registros clínicos y radiológicos de un conjunto 414 individuos ingresados correlativamente al sistema de atención clínica de la Universidad de los Andes en la ciudad de San Bernardo. Los registros que conformaron la muestra de estudio fueron evaluados respecto de su estatus de maduración esqueletal y sus características cefalométricas.

La maduración vertebral cervical aumenta con la edad, es decir, a medida que aumenta la edad también aumenta la etapa de maduración cervical. Ello es consistente con lo reportado por otros tanto en maduración cervical (Baccetti et al., 2005; Uysal \& Basciftci, 2006) como evaluando maduración en mano-muñeca (Fishman, 1982).

Los valores de las mediciones cefalométricas obtenidas en la muestra demostraron similitudes y diferencias en comparación con las normas publicadas para poblaciones caucásicas, manifestadas tanto a nivel de tejidos duros como blandos, lo cual es consistente con lo observado por otros utilizando muestras de diferentes etnias como base de comparación (Vela et al.; Hassan). El promedio del ángulo ANB $\left(4 \pm 1,8^{\circ}\right)$ se encuentra aumentado respecto a las normas caucásicas establecidas por Steiner. Esto indica que la muestra estudiada presentaría una tendencia a la Clase II esqueletal, debido a una retrusión relativa de la mandíbula. Estos valores son similares a los obtenidos por Vela et al., quienes estudiaron una muestra de 207 adolescentes de entre 13 y 12 años, Clase I dentaria, no tratados ortodóncicamente (93 estadounidenses de origen mexicano y 114 estadounidenses de origen europeo), los valores obtenidos en la muestra de sujetos estadounidenses de origen mexicano fueron de $4,6 \pm 2,1^{\circ}$, también consistentes con los obtenidos por Hassan, quien realizó un estudio en la población occidental de Arabia Saudita, con una muestra compuesta por 70 sujetos, entre 18-28 años (32 mujeres y 38 hombres), con perfiles balanceados y Clase I dentaria, donde se obtuvo como valor promedio del ángulo ANB $3,7 \pm 1,5^{\circ}$.

Se presentan a su vez diferencias entre sexos. Se observa un ángulo ANB mayor en hombres que en mujeres $\left(4,5^{\circ}\right.$ y $3,5^{\circ}$ respectivamente), indicando que los hombres del grupo de edad analizado presentan una mayor convexidad que las mujeres. Lo anterior es consistente con lo descrito previamente en estudios longitudinales, donde en eta- pas preadolescentes las mujeres pueden presentar un ángulo ANB menor que el de los hombres lo cual se revierte con posterioridad a la adolescencia conforme se manifiesta el el desarrollo facial en ambos sexos (Bishara et al., 1983). Al analizar este fenómeno en la presente muestra transversal de individuos, es importante considerar el estado de maduración cervical dado que gran parte de las mujeres se encontraban en CS4 en una etapa posterior al brote de crecimiento puberal. Por su parte, la mayoría de los hombres incluidos en este estudio se encontraban en CS2, indicando que todavía no han llegado a la cúspide del crecimiento puberal, siendo esperable que la convexidad del perfil disminuya conforme progrese el crecimiento mandibular. Esto podría explicar el menor valor del ángulo ANB en mujeres, quienes al momento del estudio ya habrían expresado en mayor medida su crecimiento mandibular en relación a los hombres. Sería esperable que con el desarrollo dicha relación se modificara, manifestando los hombres un ángulo ANB menor al de las mujeres hacia el período adulto. Lo anterior ha sido descrito en estudios longitudinales, donde el ángulo ANB disminuye de manera significativa con la edad debido a la dominancia del crecimiento mandibular por sobre el crecimiento sagital del maxilar (Bishara et al., 1983; Baccetti et al., 2009).

El promedio del valor del eje facial $\left(86,6 \pm 4,2^{\circ}\right)$ de la muestra estudiada se encuentra disminuido respecto a la norma publicada por Ricketts. El valor disminuido del eje facial indicaría una tendencia de la muestra hacia un crecimiento más vertical, no obstante conservar medidas para el ángulo SN-GoGn similares a la norma. De no mediar un tratamiento ortodóncico, no sería esperable un cambio significativo del eje facial con la edad.

Respecto a la posición de los incisivos superiores e inferiores respecto a la línea A-Po $(7,1 \pm 1,7 \mathrm{~mm}$ y $3,7 \pm 1,6$ $\mathrm{mm}$, respectivamente), se obtienen valores considerablemente mayores a los establecidos por Ricketts y Bishara (1981). Esto indica que los sujetos de la muestra presentan una biprotrusión incisiva, a pesar de presentar oclusiones normales Clase I. Esta consideración dentaria tiene una repercusión clínica directa y es relevante para la toma de decisiones terapéuticas respecto a las extracciones dentarias, al indicar que en la población Chilena mestiza joven la biprotrusión incisiva sería una característica esperable, sin necesariamente representar una indicación de tratamiento en si misma.

Del estudio de los tejidos blandos se obtuvieron diferencias entre las medidas de la muestra y la norma publicada por Legan \& Burstone. La diferencia más significativa fue la protrusión de los labios ya que la muestra tiende a valores mayores. Resultados muy similares fueron encon- 
trados por Al-Azemi et al. (2008) y Alcalde et al. (2000) en Kuwait y Japón, respectivamente. Ello justifica la valoración de los individuos según normas adecuadas para cada una de sus etnias.

De acuerdo a los datos presentados, se observa una relación entre los valores de las medidas dentarias y la de los tejidos blandos respecto a la posición de los labios. Lew (1989) estudió la influencia de la posición de los incisivos y su inclinación en la posición del labio. Al igual que en este estudio, se llegó a la conclusión de que ambos factores estaban íntimamente ligados. Se ha reportado que pacientes con protrusión bimaxilar presentan labios más protruídos (Bills et al., 2005; Lew). La protrusión labial presentada por los sujetos de la muestra guardaría una estrecha relación con la protrusión dentaria observada en la presente muestra de individuos. Ella representa un conjunto de individuos con características oclusales normales al incluir sólo pacientes con neutroclusión, resalte y sobremordida normales y ausencia de apiñamiento. Lo anterior implicaría que en población chilena infantil y juvenil de la región metropolitana de Chile, es esperable encontrar como una característica facial normal una mayor protrusión labial que la reportada por las normas cefalometricas caucásicas. Ello debe ser considerado en la ejecución de tratamientos correctivos de ortodoncia, en especial si se considera la ejecución de procedimientos que incluyan la exodoncia de premolares con la finalidad de modificar el perfil labial.

MONTT, R. J.; MIQUEL, V. M. P. \& OYONARTE, W. R. Cephalometric characteristics in young individuals with normal occlusion and harmonic profiles in Chilean population. Int. J. Morphol., 33(1):237-244, 2015.

SUMMARY: Cephalometric norms facilitate diagnosis and treatment planning in orthodontics. These are obtained primarily from adult Caucasian population. It has been suggested that these norms may vary between different ethnic groups, and there is still little information about Chilean cephalometric norms in growing individuals. A sample of 48 lateral radiographs was obtained from growing individuals ( 23 women and 25 men) with normal occlusion and harmonious profile taken between January and July 2012 at Universidad de los Andes, including 414 individuals. Radiographs were analyzed cephalometrically, obtaining soft and hard tissue values for vertical and sagittal parameters. Descriptive statistics were obtained, and differences between sexes were analyzed with student's t-test. When compared to Caucasian standards, subjects in the sample present with a Class II skeletal tendency due to a retrognathic mandible, an open facial axis, with upper and lower incisor protrusion. They also showed more protruded upper and lower lips with respect to Caucasian standards. There are differences between the cephalometric measurements observed on the individuals of the sample compared to international standards, which are evidenced at mandibular, dental and labial structures.

KEY WORDS: Cephalometric norms; Hard tissue cephalometrics; Soft tissue cephalometrics; Chilean cephalometric norms.

\section{REFERENCIAS BIBLIOGRÁFICAS}

Al-Azemi, R.; Al-Jame, B. \& Artun, J. Lateral cephalometric norms for adolescent Kuwaitis: soft tissue measurements. Med. Princ. Pract., 17(3):215-20, 2008.

Alcalde, R. E.; Jinno, T.; Orsini, M. G.; Sasaki, A.; Sugiyama, R. M. \& Matsumura, T. Soft tissue cephalometric norms in Japanese adults. Am. J. Orthod. Dentofacial Orthop., 118(1):84-9, 2000.

Baccetti, T.; Franchi, L. \& McNamara, J. A. Jr. The cervical vertebral maturation (CVM) method for the assessment of optimal treatment timing in dentofacial orthopedics. Semin. Orthod., 11(3):119-29, 2005.

Baccetti, T.; Stahl, F. \& McNamara, J. A. Jr. Dentofacial growth changes in subjects with untreated Class II malocclusion from late puberty through young adulthood. Am. J. Orthod. Dentofacial Orthop., 135(2):148-54, 2009.

Bills, D. A.; Handelman, C. S. \& BeGole, E. A. Bimaxillary dentoalveolar protrusion: traits and orthodontic correction. Angle Orthod., 75(3):333-9, 2005.
Bishara, S. E. Longitudinal cephalometric standards from 5 years of age to adulthood. Am. J. Orthod., 79(1):35-44, 1981.

Bishara, S. E.; Fahl, J. A. \& Peterson, L. C. Longitudinal changes in the ANB angle and Wits appraisal: clinical implications. Am. J. Orthod.,84(2):133-9, 1983.

Bishara, S. E. \& Fernandez, A. G. Cephalometric comparisons of the dentofacial relationships of two adolescent populations from Iowa and northern Mexico. Am. J. Orthod., 88(4):31422,1985

Fishman, L. S. Radiographic evaluation of skeletal maturation. A clinically oriented method based on hand-wrist films. Angle Orthod., 52(2):88-112, 1982.

Hassan, A. H. Cephalometric norms for saudi adults living in the western region of Saudi Arabia. Angle Orthod., 76(1):109-13, 2006.

Jacobson, A. The "Wits" appraisal of jaw disharmony. Am. J. Orthod., 67(2):125-38, 1975. 
Jain, P. \& Kalra, J. P. Soft tissue cephalometric norms for a North Indian population group using Legan and Burstone analysis. Int. J. Oral Maxillofac. Surg., 40(3):255-9, 2011.

Legan, H. L. \& Burstone, C. J. Soft tissue cephalometric analysis for orthognathic surgery. J. Oral Surg., 38(10):744-51, 1980.

Lew, K. Profile changes following orthodontic treatment of bimaxillary protrusion in adults with the Begg appliance. Eur. J. Orthod., 11(4):375-81, 1989.

Mamandras, A. H. Linear changes of the maxillary and mandibular lips. Am. J. Orthod. Dentofacial Orthop., 94(5):405-10, 1988.

McNamara, J. A. Jr. A method of cephalometric evaluation. Am. J. Orthod., 86(6):449-69, 1984.

Ricketts, R. M. A foundation for cephalometric communication. Am. J. Orthod., 46(5):330-57, 1960.

Scavone, H.; Zahn-Silva, W.; do Valle-Corotti, K. M. \& Nahás, A. C. Soft tissue profile in white Brazilian adults with normal occlusions and well-balanced faces. Angle Orthod., 78(1):5863, 2008 .

Soto, Q. L. \& Tapia, C. R. Diagnóstico Nacional de Salud Bucal del Adolescente de 12 años y Evaluación del Grado de Cumplimiento de los Objetivos Sanitarios de Salud Bucal 20002010. Santiago de Chile, Facultad de Odontología Universidad Mayor, MINSAL-CONICYT, 2007

Steiner, C. C. Cephalometric for you and me. Am. J. Orthod. Dentofacial. Orthop., 39(10):729-55, 1953.

Swlerenga, D.; Oesterle, L. J. \& Messersmith, M. L. Cephalometric values for adult Mexican-Americans. Am. J. Orthod. Dentofacial Orthop., 106(2):146-55, 1994.

Tweed, C. H. The Frankfort-mandibular plane angle in orthodontic diagnosis, classification, treatment planning, and prognosis. Am. J. Orthod. Oral Surg., 32:175-230, 1946.

Uribe Restrepo, G. A. Ortodoncia: Teoría y Clínica. Medellín, Corporación para Investigaciones Biológicas, 2010.

Uysal, T.; Ramoglu, S. I.; Basciftci, F. A. \& Sari, Z. Chronologic age and skeletal maturation of the cervical vertebrae and handwrist: is there a relationship? Am. J. Orthod. Dentofacial Orthop., 130(5):622-8, 2006.

Vela, E.; Taylor, R. W.; Campbell, P. M. \& Buschang, P. H. Differences in craniofacial and dental characteristics of adolescent Mexican Americans and European Americans. Am. J. Orthod. Dentofacial Orthop., 140(6):839-47, 2011.

\author{
Dirección para Correspondencia: \\ Dr. Rodrigo Oyonarte Weldt \\ Cirujano-Dentista, Ortodoncista \\ Profesor Asociado \\ Facultad de Odontología \\ Universidad de los Andes \\ Mons. Álvaro del Portillo 12.455 \\ Las Condes, Santiago \\ CHILE
}

Email: royonarte@miuandes.cl

Recibido : 15-05-2014

Aceptado: 14-01-2015 